\title{
E'S-AODV: An Energy Efficient Multipronged Reliable Strategy ensuring Secure and Scalable QoS over Disaster Response Applications
}

\author{
Karan Singh ${ }^{1}$, Rajeev Gupta ${ }^{2}$ \\ ${ }^{1}$ M.M. Institute of Computer Technology and Business Management, MMDU, Mullana - India, \\ karanola123@gmail.com \\ ${ }^{2}$ Departement of Computer Science \& Engineering, M. M. Engineering College, MMDU, Mullana - India. \\ rajeev.gupta@mmumullana.org
}

\begin{abstract}
Mobile Ad-hoc Network is a group of wireless mobile nodes without fixed infrastructure and each node can work as both a router and host. Devices can move randomly and set-up a network that is free from central infrastructure with mobility in nature. Network issues are solved by various routing protocols. Many "Quality of Services (QoS)" must be considered in constructing routing protocol. AODV based on-demand routing is most widely used protocol in MANET. In on-demand based routing routes breaks due to the node mobility and less battery power. Recent work on MANET protocols have focused on achieving these characteristics which suit one specific networking scenario. There is no existing specific protocol that can work well in all multipronged networking parameters. This paper proposed a protocol $\mathrm{E}^{2} \mathrm{~S}-\mathrm{AODV}$ that will reduce path breakage due to battery drains. Further reliability, scalability, secured route with QoS, minimum control overhead and energy consumption will be simulated.
\end{abstract}

Key words: Energy Efficiency, E²S-AODV, MANET, QoS Matrices.

\section{INTRODUCTION}

A mobile ad-hoc network (MANET) is a self-organizing wireless network made up of moving mobile nodes and requires no fixed infrastructure; these types of networks have to follow some precise rules called "protocols". Wireless networks can be of two types:

- Infrastructure Based Network: A set-up requiring integrated and permanent gateways.

- Infrastructure-less or Ad-Hoc Network: A set-up lacking any type of centralized gateways.

Term Ad-hoc says about a system that can modify itself according to topology and in terms of device used. These used devices can communicate with other device within same network, move independently within network and can make up themselves accordingly [1]. The term mobile ad-hoc network involves three diverse technical words: (i) Mobile i.e., travelling independently in nature, (ii) Ad-hoc means tiny time duration, (iii) Network i.e., pool of several nodes [2]. Every node that actively participating in network works as switch as well as host and under this relationship the organization should interchange packets among different hubs.

Various applications like weather observation, tracking, tsunamis, wildfire and emergency disaster rescue and prevention operations, underwater level navigation can be outperform by MANET because of its temporary establishment nature. These types of networks are popular for data communication due to its quick environment set-up with minimum resources. MANET can solve various problematic situations like flood, drought, fire in forest, tsunamis, underwater movements in oceans, emergency rescue operations, disaster prevention operations, weather forecasting, tracking of wild animals, traffic controlling and vehicle movement tracking and message passing on highways [2]. Thus, these networks can be appropriate and usable for solving these types of situations in current trends and in future also.

While making an ad-hoc system it is appropriate to select ondemand protocols like AODV (Ad Hoc On-Demand Distance Vector) because of its high mobility, less control overhead, ondemand resource nature is needed. While setting up ad-hoc network in on-demand based protocol some parameters are more critical i.e. link establishment, optimal route selection, data transfer rate, successful data transmission rate at receiver, security issues, power consumption and utilization of available limited resources etc.

Data transmission from source to destination have to take care of various issues like packets should reach through a shortest path i.e. optimal path, received data packet should be secure form various security attacks, reliable in nature, delay should be minimum while transmission, participating node/link should aware from node/link failure due to energy drain etc. So, data transmission should take care of all these parameters "to be published" [3]. In order to transmit data in MANET we need effective protocols and all these protocols should meet some desired matrices i.e. PDR, throughput and delay. Through these metrics following network parameters can be explained [4]-[6]. 
Reliability metrics make sure that the MANET can transmit data successfully to its destination through selected path. Reliability metrics is directly depends on various network activities like link failure, node failure, various security attacks etc.

Scalability metrics make sure that the network can perform efficiently whenever number of participating nodes increases i.e. network size grows. Scalability metrics directly depends on number of nodes (N), network density (D), number of links (L) etc.

Power consumption (energy used by node) metrics can be used for calculation of various energy parameters like energy used by node, total network energy, link energy, energy loss of link and node while transmission, remaining node energy after successful transmission, identification of failure node due to energy drain etc.

Delay metrics used for calculating the time taken for a data packet to be transmitted across the link from source node to destination node. End-to-end delay depends on some factors like packet size, route length, hop count in link etc.

Packet Delivery Ration (PDR) is a parameter of quality of service $(\mathrm{QoS})$ that defines the network performance in respect of total packet sent by source (TPss) vs. packet delivered at receiver (TPrr) [2]. PDR of any protocol should be as high as possible for best performance "to be published" [3].

$\mathrm{PDR} \%=(\mathrm{TPss} / \mathrm{TPrr}) * 100$.

Throughput metrics (Tpt) is the ratio of the total packets that reaches successfully to a receiver from the sender [2], "to be published" [3].

$\mathrm{Tpt}=\frac{\text { No. of packet delivered } * \text { packet size in bits }}{\text { Total Simulation Duration }}$

To achieve these issues in a better way a solution named $E^{2} S-$ AODV (Energy Efficient Secured Ad-hoc On-demand Distance Vector) routing protocol is introduced. The proposed protocol is an enhanced version of traditional AODV with improvement in security, optimality and energy consumption. Security and authentication of "hello" message authors used "Lightweight digital watermarking (LDW)" [6]. LDW works like a data hiding method that decreases computation cost over traditional cryptography methods. Event messages are secured with CST (Ciphertext Stealing Technique) and encrypt it with quVanstone ECC based public key cryptography [6], [7]. In the end, a procedure named Energy_Efficient_routeDiscovery() will calculate the residual energy of nodes that will decide which node have sufficient energy to take part in communication [8].

$\mathrm{E}^{2} \mathrm{~S}-\mathrm{AODV}$ can be a multipronged solution of many disasters like situations like flood, drought, fire in forest, tsunamis, underwater movements in oceans, emergency rescue operations, disaster prevention operations, weather forecasting, tracking of wild animals, traffic controlling and vehicle movement tracking and message passing from one moving vehicle to other on highways etc "to be published" [3].

For evaluate results of various metrics an open source tool Network Simulator Version 2 (NS2) is used. NS2 deals with TCL as scripting language. TCL based scripts are specially designed for simulation purpose in research area of communication network.

Paper is organized as follows: - Section 2 covers some related prior work about energy efficiency, security and optimality in recent years and finds research gaps. Segment 3 describes the research methodology to be followed in whole research journey. Section 4 identifies the problem, formulates it and proposed solution also discussed in this section. Section 5 describes simulation setup. Section 6 elaborates results and performance evaluation of proposed in respect of several multipronged matrices. This section also compared the results of existing and proposed $E^{2} S$-AODV. Section 7 concludes about overall efforts made in formulation of the research. In last, segment 8 provides future directions.

\section{RELATED WORK AND RESEARCH GAP}

Several efforts were made to improve various quality assurance metrics of ad-hoc protocol's (specifically AODV) i.e. throughput, packet delivery ration, delay in message delivery, optimization of ad-hoc route, secured message transmission, minimum overhead, energy efficient transmission, reliable and scalable network selection etc.

Authors have used an approach that will change the frequency transmission of packets and alertness of mobility in a reactive based AODV protocol. Authors focus on the victim node that will not waste its resources while encountering malicious packets. The goal is to improve the routing process by avoiding the resource consumption attack [9].

Authors told about various methods that can identify many parameters to achieve a trustworthy route i.e. node residual energy, link termination time, link obtainable time, stability of nodes, node successful data communication, received signal strength etc. Authors proposed a Mobile Agents based Reliable and Energy Efficient Routing Protocol (MAREERP) to accomplish two parameters one as reliability and other one is energy efficiency in MANET [10].

The reliability of MANET is achieved by finding out of critical links of a network. Authors reduced the calculation efforts of complexity related to reliability and made it practical for scalability purpose for large scale networks [11].

Author mentioned some decisions that will provide reliability to highly dynamic MANETs by combining two different metrics. These decisions will work in multi hop ad hoc environment. The proposed protocol binds two properties of ad hoc network to achieve this goal; one is RSS (Received Signal Strength) with SINR. The resulting new routing scheme is called as Link Quality Aware Ad-hoc On-Demand Distance 
Vector (LQA-AODV) routing. This type of protocol can rapidly adjust to lively changes in network arrangement and improved the reliability parameter of MANETs by improving routing decisions [12].

Authors' projected an algorithm named as UGFT i.e. Universal Generating Function (UGFT). UGFT based algorithm works on various ways that will focus on minimal path and minimal cut. UGFT will provide the reliability to network while using CGSR protocol. Author defined reliability as a probability metric that says about the successful transmission of message from the starting place to destination palace without any delay. The UGFT uses algebraic procedures that will find performance distribution of whole system. The proposed Universal Generating Function (UGFT) in CGSR is the first method that calculates the MANET reliability through probability [13].

Authors advised a method that will reflect on the impact of a variety of mobility models like RWPM, GMMM etc. regarding reliable MANET. Authors changed the position of node randomly and found that results are same as that of MANET by considering node movements using RWPM and GMMM further authors also analyzed the results of the network reliability with random mobility network and without mobility network. The technique is easy and efficient with minimum number of random variables [13], [14].

Authors concluded about scalability metric of homogenous and heterogeneous network. Scalable property will work practically well homogenous system environment for all protocols but their performance suffer drastically over heterogonous system environment. Authors concluded about scalability metric of homogenous and heterogeneous network. Scalable property will work practically well homogenous system environment for all protocols but their performance suffer drastically over heterogonous system environment. Scalable performance of AODV, OLSR and DSR are compared. AODV performs better scalability for both homogeneous and heterogeneous network was also concluded [15].

Authors presented a new model based on trust metric and evaluated how well a protected node can be planted in a MANETs through QoS metrics. This trust method can enhance overall network performance by minimizing packet loss, increasing throughput and reducing power use while encountering various malicious black hole nodes. Proposed trust model uses different trust values i.e. direct, indirect and mutual trust values. These values will reflect the behavior of sensor nodes. The mixture of these trust values can calculate the trust level of autonomous sensor nodes and gives the identification of an anomalous node in the network [16].

Authors planned a technique Fuzzy Logic Controller (FLC) based routing. FLC based this method produced a routing algorithm "Neuro Fuzzy Based Dynamic Secure Routing (NFBDSR)". Route metric will be calculate by some variable i.e. (i) left over (residual) energy, (ii) processing potential of node, (iii) available bandwidth, (iv) mobility of node and (v) trust value of node. The NFBDSR routing algorithm identifies suspect full node and secures network from different types of threats and attacks [17].

Authors discussed many QoS metrics based routing protocol named as A-OLSR. A-OLSR absorbs the profit of OLSR protocol and showed that A-OLSR performs appreciably better in terms of QoS metrics i.e. PDR, Throughput, Energy Consumption and Delay [18].

Authors discussed about a multipath routing for MANET named as PSOGSA (Particle swarm optimization-genetic search algorithm). PSOGSA is a secure and QoS aware protocol for mobile ad-hoc networks. Optimal path is chosen by a search based algorithm "cuckoo search". Proposed algorithm is used to pick power efficient multipath routes from source node to destination node. This power efficient based protocol will also help to get rid of attackers or selfish nodes from the system [17]-[19].

Authors compared Energy Efficient Multicast Routing Protocol for MANET with Minimum Control Overhead (EEMPMO) with MAODV minimized the problem of delay because of the directional diffused forwarding routing and also Network segmentation problems occur when a link error arises due to primary root failure. And as a result, the need for maintenance and reconstruction of tree was also minimized by backup roots [20].

Authors projected a Low Overhead Localized Flooding (LOLF). Proposed protocol is based on some technical points i.e. (i) query localization, (ii) query localization optimization. These points will minimize routing overhead of traditional AODV protocol. While local repairing procedure proposed protocol have to calculate node/hop count information for all nodes. Proposed work will control the spread of packets in path discovery and path repair mechanism while incurring only a small increase in the size of control information in the packet [21].

Authors presented an energy conservation technique named LBTC. Full name of LBTC is Location Based Topology Control that coordinates with SS (Sleep Scheduling) for ad-hoc networks. LBTC works on two parameters i.e. (i) topology control of ad-hoc system and (ii) energy management parameters. The nodes that are not part of established communication link are kept in sleep state for energy saving purpose. Information of neighborhood node location will help in reducing transmission power of a node. Sleep state of a node is selected on the behalf of traffic conditions. In the projected proposal, a node goes to sleep state only when its nonexistence does not produce any type of local partition in its neighborhood [22]. Authors presented a solution and found that energy utilization and throughput in small size MANET do not produce any major difference. The proposed solution named as "Efficient Power Aware Routing". Further, AODV performs 
better over DSR for medium and large MANETs. In small size MANET; the performance of EPAR, MTPR and DSR was compared on behalf of various metrics. In energy used conditions while communication, AODV performs reasonably well over EPAR and MTPR in medium and large size MANETs [23].

Authors proposed an enhanced version of AODV named as AOMDV-ER (Energy Reduction Multipath Routing Protocol using Recoil Technique). Proposed protocol outperforms the some existing protocols like AODV, AOMR-LM, AOMDV and SRMP. Proposed protocol will improve various QoS metrics like PDR, power utilization, routing overhead, network lifetime etc [24].

Authors presented a new approach which fundamentally depends on power quality of movable nodes and their mobility. Proposed method will upgrade the OLSR protocol. Author attempts the power utilization technique with the help of genetic algorithm optimization technique [25].

Some routing protocols i.e. DSDV, DSR against AODV were compared and find that AODV performs better as compare to other protocols due to its easily adaptable mobility nature and energy efficiency [2]. Thus, AODV on-demand based suits well to explore various research issue related to MANET i.e. security, energy efficiency, optimality etc. Many fact finding previous work and attempts in these directions are elaborated in above paragraphs. Two critical issues are identified i.e. energy efficiency in data transmission and secure communication. Both issues can be picked up to study over any on-demand based traditional protocol. Energy Efficient with Secured Reliable Routing Protocol (EESRRP) for MANETs and compared with AODV [26]. Due to missing the multipronged achievement of metrics and their effect on network; a novel security technique and a new energy efficient procedure named $E^{2} S-A O D V$ will fulfill these critical research gaps.

\section{RESEARCH METHODOLOGY}

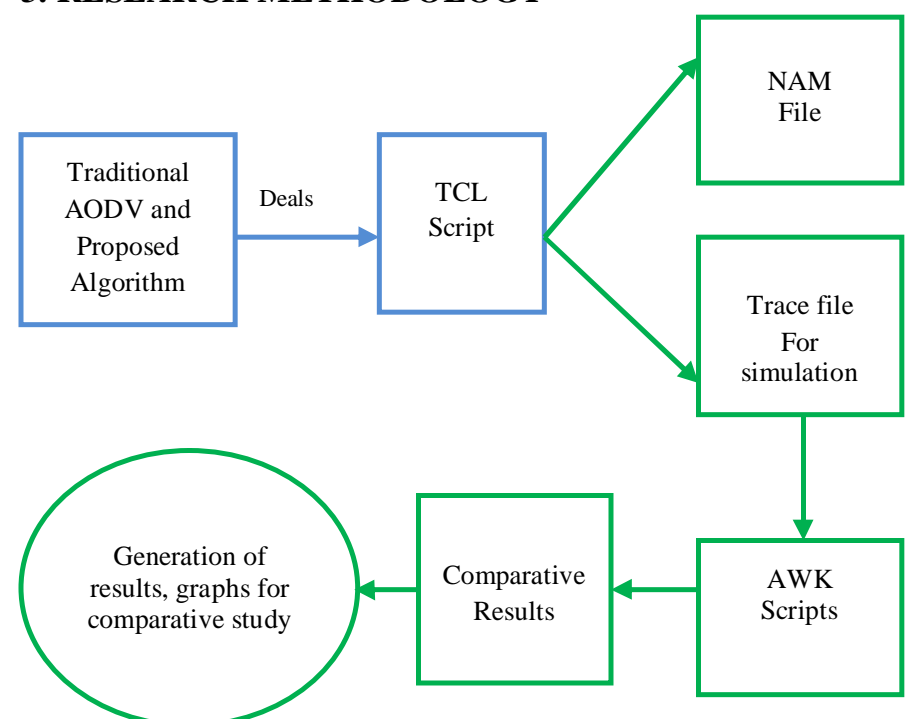

Figure 1: Research methodology for $E^{2} S-A O D V$
Figure 1 shows the methodology used in proposed $\mathrm{E}^{2} \mathrm{~S}-\mathrm{AODV}$. A TCL (Tool Command Language) script is formed that will focus on network traffic scenario and wireless conditions of various mobile nodes. This TCL script will be operate on a network simulator i.e. NS2. TCL is a text file having TCL scripts. TCL is an open source language used for building application on Linux like operating systems. While simulating, TCL file will make a "trace file" and the "NAMfile". NAM is a TCL centered simulation tool that can envision the NS2 simulations and real world packet data transmission. The first step to use NAM is to produce a NAM trace file. NAM carries some useful information about nodes, links, queues, node connectivity and packet trace information. Trace file generates "AWK script". AWK will generate results and analyzing the purposed protocol. AWK works as a scripting language and used for manipulating data and generating reports.

\section{PROBLEM DEFINITION AND PROPOSED PROTOCOL (E' ${ }^{2}$ S-AODV)}

There are many on demand based reactive routing protocols, among them AODV is better to choose for diagnose about various MANET issues. AODV have better mobility in nature and energy awareness capabilities as compare to other reactive protocols thus authors are enriching this traditional protocol to construct upon.

MANET have some characteristics i.e. dynamic topology, open access to lively nodes, lack of dominant monitoring, that are likely to be secure from various serious security threats or attacks. Therefore, secure communication is an important aspect and opportunity in research. Various protocols are designed for secure data transmission among mobile nodes like AODV, DSDV and DSR etc. AODV performs two-step process for communication: (i) route searching, and (ii) route maintenance operations for communication. Optimal route selection is very important part of routing process. Optimal path cam be selected on behalf of some network parameters like minimum hop count in path, minimum resource count in path, minimum energy consumption in path etc. Formerly many research efforts was attempted to fulfill this task with the help of ACO (Ant Colony Optimization), Enhanced-ACO, Swarm based Intelligence (SI) etc. These types of efforts elaborated and proved that swarm based routing techniques will provide optimality, reliability and efficiency of MANETs and also reduce control overhead due to their scalable nature. Further selection of a reliable path that can exclude critical energy drain nodes from selecting in communication link will increase reliability and optimality of network. AODV initially was introduced without considering security issues during message transmission [27]. Noteworthy efforts have been completed to secure AODV protocol in ad-hoc network but there are still serious challenges to defeat [28], [29].

After going through few secured protocols of some former studies, an improved protocol named $\mathrm{E}^{2} \mathrm{~S}-\mathrm{AODV}$ is proposed to improve the security and optimality parameters of AODV. $\mathrm{E}^{2} \mathrm{~S}$-AODV selects optimum path by calculating residual 
Karan Singh et al., International Journal of Advanced Trends in Computer Science and Engineering, 9(4), July - August 2020,5933 - 5941

energy technique i.e. nodes having sufficient residual energy can participate in communication link this leads to optimality. Hello messages broadcasted periodically to inform its neighbors that the connection to the host is active. LDW technique authenticates these hello messages. In the end, original event message was encrypted with CST (Ciphertext Stealing Technique) and qu-Vanstone ECC based public key cryptography [30], [31]. This segment also elaborates in detail about proposed effort. Objective of this effort is to recommend an optimized and secure ad-doc on-demand type protocol that can work for multipronged parameters in MANET.

\subsection{Proposed Algorithm: $E^{2}$ S-AODV (Energy Efficient Secure Ad-hoc On-demand Distance Vector)}

Step 1: Set initial_energy $=100$ joules

Step 2: Each nodes of network periodically broadcasts "hello" messages to neighboring nodes via "Lightweight Digital Watermarking Technique (LDW)".

Step 3: Sender broadcasts RREQ for finding optimal route to destination and secure broadcasted message with CST \& qVECC based asymmetric cryptography.

Step 4: Calculate residual energy of nodes

IF (Residual energy $>=$ Threshold energy (i.e. $(1 / 2) *$ initial_energy))

Node is selected for active route formation ELSE

Energy_Efficient_route_Discovery(); procedure start

Step 5: Neighbor node receives RREQ

IF (Neighbor is Destination)

Destination decrypts the message with public key to ensure authenticity of sender node and then sends Route Reply (RREP) to source.

ELSE

Forward RREQ packet to its adjacent nodes until destination is reached

Step 6: Destination reply with RREP and source node receives multiple RREP with different sequence number.

Step 7: Sender accepts the optimal route with latest sequence number and having higher level of residual energy of route.

\section{SIMULATION ENVIRONMENT SETUP}

Simulation parameters are enlisted in table 1. A network simulator NS-2.34 is used to perform metrics evaluation. Simulator is constructed in ubuntu operating system environment. Several quality assurance metrics i.e. packet delivery ration, throughput and end-to-end delay are analyzed. Comparison graphs between AODV and proposed protocol are generated related to these quality assurance metrics.
Table 1: Simulation Parameters

\begin{tabular}{|c|c|}
\hline Simulation & Parameter Values \\
\hline Simulator & NS-2.34 \\
\hline MAC Type & 802.11 \\
\hline Channel & Wireless Channel \\
\hline Antenna Type & Omni-directional \\
\hline Radio Propagation & Two-Ray Ground \\
\hline Interface Queue & DropTailPriQueue \\
\hline Simulation Area & $1000 \mathrm{~m}^{*} 1000 \mathrm{~m}$ \\
\hline Mobile Nodes & $25,50,75,100,125,150$ \\
\hline Pause Time & $0 \mathrm{~s}$ \\
\hline Speed & $1.0,2.0,5.0,7.0,10.0 \mathrm{~m} / \mathrm{s}$ \\
\hline No. of Connections & 6 (25 nodes); $13(50$ nodes $) ;$ \\
& 20 (75 nodes); $26(100$ \\
& nodes $) ; 33(125$ nodes $) ; 41$ \\
& GA-CARP, E ${ }^{2}$ S-AODV \\
\hline Routing Protocols & TCP \\
\hline Traffic Sources & 500 sec. \\
\hline Simulation Time & Throughput \\
\hline Performance Metrics & PDR, end-to-end delay, \\
\hline
\end{tabular}

6. SIMULATION RESULTS AND COMPARATIVE ANALYSIS OF MULTIPRONGED PERFORMANCE MATRICES

Purpose of the research is to design a reliable algorithm for MANET communication that ensures security, stability \& quality of service with energy conservation over MANET communication. It is clear from the below figures that objective of the research is achieved i.e. our proposed protocol $\mathrm{E}^{2} \mathrm{~S}-\mathrm{AODV}$ outperforms existing routing protocol. Figure 2 depicts the PDR with resects to number of nodes. Initially PDR is high for lesser number of nodes but as we increase number of nodes PDR decreases \& showing a continuous declining trend. Despite this $E^{2} S-A O D V$ outperforms the existing one for every node. Figure 3 shows Delay with respect to number of nodes. Initially Delay is low for lesser number of nodes but figure shows an increasing trend with the increase in the number of nodes. Delay shows rising trend up to 100 nodes then shows a dip for 125 nodes then again increasing with increase number of nodes. This issue is still under research. But $\mathrm{E}^{2} \mathrm{~S}-\mathrm{AODV}$ outperforms existing protocol. 


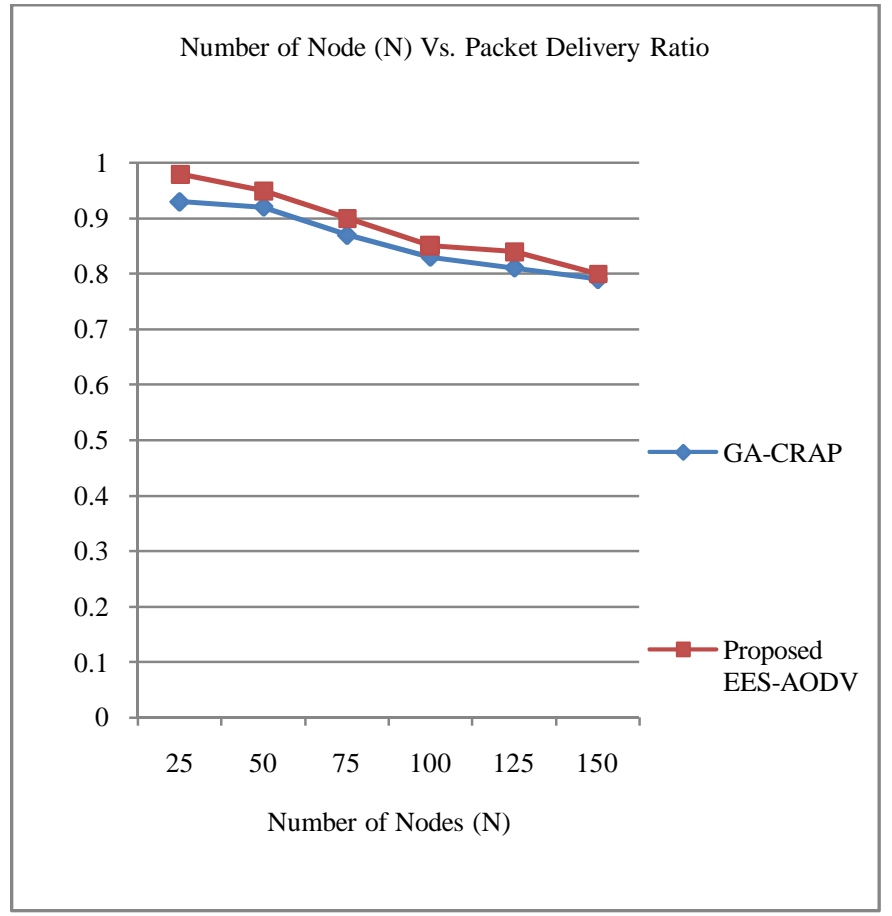

Figure 2: Number of Node (N) Vs. PDR Graph.

Figure 4 depicts throughput with respect to number of nodes. Proposed algorithm always outperforms existing one \& throughput in case of proposed algorithm is much better than the existing one. Throughput in proposed algorithm shows a fluctuating trend. Throughput decreases with increase in number of nodes due to more routing process. Hence overall performance of the proposed algorithm is much better than the existing one in terms of PDR, Throughput, and Delay. The proposed algorithm also ensures better security of the network and protects the network from various attacks like Sybil attack etc. It also conserves energy of the networks that leads to lifetime of nodes that in turns leads to better stability of the MANET.

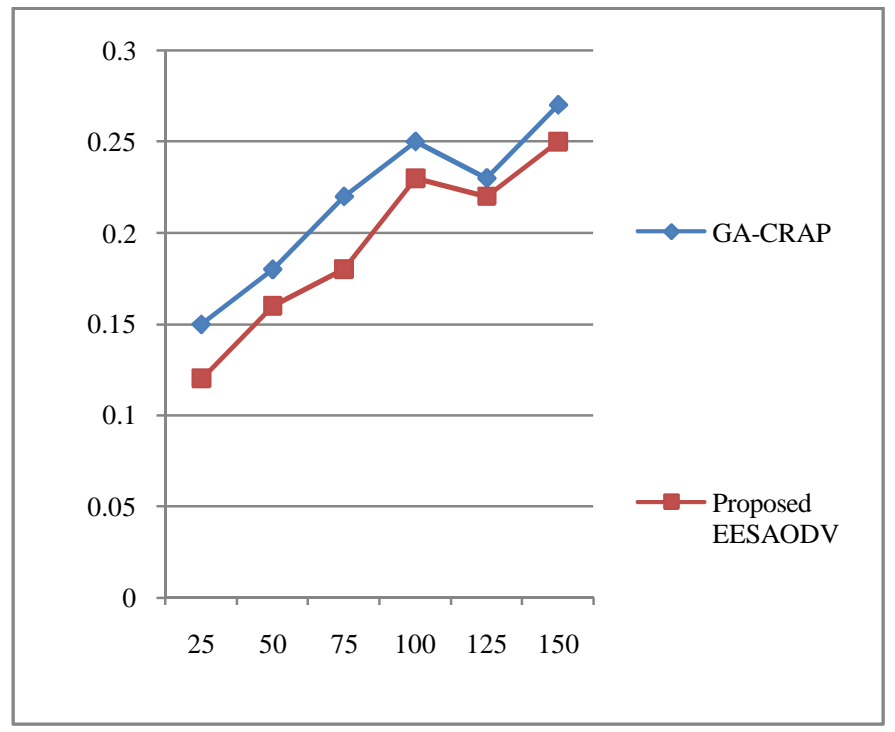

Figure 3: Number of Nodes (N) Vs. Delay Graph.

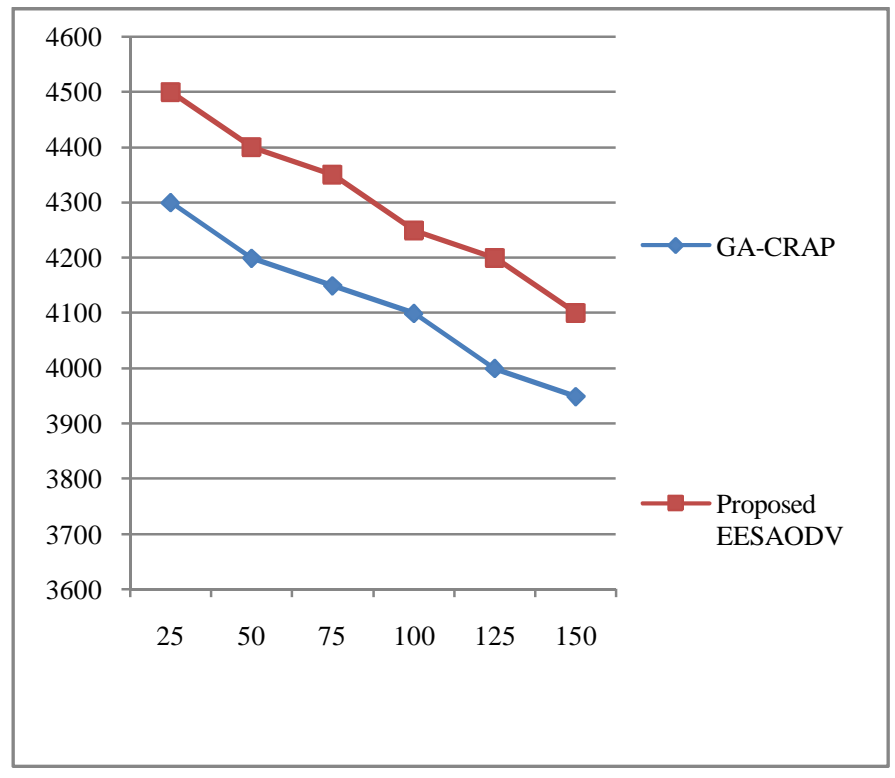

Figure 4: Number of Nodes Vs. Throughput Graph

Therefore, in order to provide efficient communication as well as to minimize the power consumption and transmission overhead, reliable routing protocols play an important role in MANET. On behalf of above discussion and graphs various issues i.e. reliability, scalability, end-to-end delay, control overhead, QoS support, Power consumption etc can be achieved for MANETs.

Next sub-sections from 6.1 to 6.5 will define some multipronged trends that can be analyzed on the basis of simulated graphs; these multidimensional parameters are as follows:

\subsection{Reliability}

The reliability of MANET is defined as the probability that a message converted from the source can be successfully passed through the MANET and reached the target. Reliability can be affected by various network activities like dynamic topological changes, security attacks, node failure due to low energy, link failure [32],[33]. Reliability issues can be explained by various parameters like choosing minimum time consumption in transmission, secure message transmission, removing failure node from link etc [13]. In this paper two reliability factors secure transmission of message and node failure due to less power are solved, security of message by using cryptography and node failure by using energy model $E^{2} S-A O D V$.

\subsection{Scalability}

Scalability is the ability of a routing protocol when any MANET metrics performs efficiently corresponding to the growing nature of network. Various MANET properties like PDR, throughput, power consumption etc. can be checked for scalability purpose [15]. Various scalability parameters for MANET that can affect performance of any protocol are number of nodes in a network, total number of links, average movement of movable nodes (average mobility rate), node 
density, frequency of established link and average number of simultaneous connections. Many ad hoc metrics like PDR, throughput, delay can be analyzed on the basis of growing nature of ad hoc network [33]. $\mathrm{E}^{2} \mathrm{~S}-\mathrm{AODV}$ protocol is working well for all metrics from initial setup of 25 nodes and varies up to 150 nodes in our simulation environment; therefore one important multi-prong dimension scalability can be achieved.

\subsection{Minimum control overhead}

The control packets sent by network nodes for each data packet is called the routing load of the network. Control packet consists of request, route reply and route error. Some network parameters are affected by these control packets like bandwidth utilization, consumed resources and available energy while data transmission. For best use of bandwidth any routing protocol should transmit minimum control message for need for transmission operation. Therefore, protocols should be aware about threshold value of control message and should be comparatively low further this leads to conserve battery power [34], [35]. E $\mathrm{E}^{2} \mathrm{~S}-\mathrm{AODV}$ protocol shows better end-to-end delay results as compare to traditional one which leads to a theory of minimum control message generation and transfer over MANET; therefore minimum control overhead is achieved.

\subsection{Quality of Service (QoS) Support}

There is no exact description for Quality of Service which is accepted universally. But we can say that the network which is efficient to give some guarantees about the services is QoS network [32]. Routing protocol should integrate with some quality services which further leads to usefulness of network. The ultimate purpose of providing QoS is to attain a more expected behavior of network, thus information is transmitted correctly by the network and also the resources are utilized in a better manner [35]. QoS is different from shortest path selection because shortest path may reach to the target earlier but might be using more resources; Whereas, QoS metric may not be the shortest path but it can be the most reasonable path with minimum consumption of network resources (bandwidth, power). QoS aware routing protocol must achieve some constraints that should be satisfied i.e. path selection, estimated resources, route detection, resource reservation, route maintenance etc. In general, the level of service depends on some parameters, known as QoS parameters i.e. bandwidth availability, end-to-end delay, delay variations, loss rate of packets etc [36]. A major benefit of this protocol is that more precise end-to-end delay can be achieved with minimum use of resources.

\subsection{Power consumption}

Laptops, mobiles, PDAs, thin clients, palmtops etc. can be used as mobility nodes in MANETs. One major issue i.e. limited battery power is there that can affect performance of these devices [8]. A node consumes power in variety of activities like in data transmission, while listening to the network for any possible data transmission request, in idle state etc [33]. Many other energy drain based causes are like collision, larger packet size, channel sensing in idle state, overhearing, higher bit rate, message flooding and dynamic topology etc. One important attribute of energy is residual energy. Residual energy can be defined as left over energy of any node before drain. Residual energy is directly proportional to network performance. So, maximizing the usages of energy and lifetime of batteries of each node and entire MANET is very critical task [37]. $\mathrm{E}^{2} \mathrm{~S}$ AODV protocol selects the optimal path which has sufficient residual energy to complete the communication process. Thus established link will be reliable in terms of available energy and residual energy will be used efficiently in data transmission. Critical nodes cannot take part in communication path. In this way $E^{2} S-A O D V$ uses energy in efficient way in data transmission.

\section{CONCLUSION}

In this paper a new protocol $\mathrm{E}^{2} \mathrm{~S}-\mathrm{AODV}$ is proposed for energy efficient and multipronged reliable strategy ensuring secure and scalable QoS over MANETs. Traditional AODV, EESRRP and GA-CRAP were lacking behind and multipronged reliable, secure and energy efficiency while message transmission. Therefore, both these parameters are fulfilled. Residual energy is used for optimality (efficient route selection through energy). "Hello" message for node are authenticated with LDW. Event messages are transferred using CST with qu-Vanstone ECC based public key cryptography. In a simulation environment, proposed protocol is compared with existing and analyzed for some multipronged reliable parameters ensuring secure and scalable QoS over MANETs. Proposed protocol's performance is better than conventional and can work with multidimensional matrices. Simulation results of $E^{2} S-A O D V$ achieved significant betterment in terms of end-to-end delay, packet delivery ration and in throughput further this betterment leads to improve some multipronged parameters of MANET like reliability, scalability, QoS, control overhead etc. Hence proposed protocol performs better than existing.

\section{FUTURE SCOPE}

In future, $E^{2} S-A O D V$ can be test with any disaster response applications. All available protocols are open for advancement and enhancement so that new methodologies can be applied for betterment. The scope in $E^{2} S-A O D V$ is also open for enhancement on various MANET fields.

\section{REFERENCES}

1. K. Singh, and A. K. Yadav. A Qualitative and Quantitative Analysis of Routing Protocols in MANET, International Journal of Advanced Research in Computer and Communication Engineering, 5(7), 2016.

2. K. Singh, and R. Gupta. Comparative Assessment and Performance Analysis of Numerous Mobile Ad-Hoc Network Routing Protocols, Journal of Computational and Theoretical Nanoscience, Vol. 16, pp. 3906-3911, 2019. 
3. K. Singh, and R. Gupta. SO-AODV: A Secure and Optimized Ad-Hoc On-Demand Distance Vector Routing Protocol over AODV with Quality Metrics for Disaster Response Applications, To be Published by IGI global publications, US, 2020.

4. K. V. Arya, and K. N. Tripathi. Power aware and secure routing in mobile and ad-hoc networks, IEEE 8th International Conference on Industrial and Information Systems, Peradeniya, pp. 477-482, doi: 10.1109/ICIInfS.2013.6732031, 2013.

5. S. Bhatia, B. Radim, and M. Kevin. Poster Abstract: Throughput-Delay Tradeoff in Small and Sparse Mobile Ad hoc Networks, 2019.

6. A. K. Yadav, and A. Kush. E' -SCRP: An Energy Efficient Secure Cluster based Routing Protocol for 3D Underwater Acoustic MANET (UWMANET), International Journal of Innovative Technology and Exploring Engineering, ISSN: 2278-3075, 8(11), 2019. https://doi.org/10.35940/ijitee.K1391.0981119

7. D. M. Kuryazov. Algorithm for ensuring message confidentiality using elliptic curves, International Journal of Advanced Trends in Computer Science and Engineering. Volume 9, No.1, Jan. - Feb. 2020. https://doi.org/10.30534/ijatcse/2020/44912020

8. N. Kamboj, Dalip, and M. Rai. An Enhanced Energy Efficient Secure Routing Protocol for MANET, International Journal of Advanced Science and Technology, Vol. 29, No. 05, pp. 1135-1142, 2020.

9. H. Rachid, and M. Ouzzif. Secure And Reliable Routing In Mobile Adhoc Networks, International Journal of Computer Science \& Engineering Survey (IJCSES), Vol.3, No.1, 2012. https://doi.org/10.5121/ijcses.2012.3105

10. S. Nallusamy, S. Appavupillai, and S. Ponnusamy. Mobile Agents based Reliable and Energy Efficient Routing Protocol for MANET, International Journal of Intelligent Engineering and Systems, 9, pp. 110-116. 10.22266/ ijies2016 .0930.11, 2016.

11. K. S. Meena, and T. Vasanthi. Reliability Analysis of Mobile Ad Hoc Networks Using Universal Generating Function, Quality and Reliability Engineering International, 32, 10.1002 / qre.1731, 2014.

12. M. Sangman. Link Quality Aware Route Discovery for Robust Routing and High Performance in Mobile Ad Hoc Networks, pp. 281-288, 10.1109/ HPCC. 2009.71, 2009.

13. N. Padmavathya, and S. K. Chaturvedib. Reliability Evaluation of Mobile Ad Hoc Network: With and Without Mobility Considerations, International Conference on Information and Communication Technologies (ICICT 2014), 2014.

14. S. Choudhary, and S. Jain. A survey of energy-efficient fair routing in MANET, International Journal of Scientific Research in Science, Engineering and Technology, vol. 1, pp. 416-421, 2015.

15. M. Singh, and A. Singh. Performance Evaluation of MANET Routing Protocols with Scalability for HTTP Network Load Using OPNET Modeler, International
Journal of software \& Hardware Research in Engineering, Volume 1 Issue 2, Page 25, ISSN NO: 2347-4890, 2013.

16. J. Manoranjini, A. Chandrasekar, and S. Jothi. Improved QoS and avoidance of black hole attacks in MANET using trust detection framework, Automatika, 1-11. 10.1080/ 00051144. 2019. 1576965, 2019.

17. S. Sahu, and S. Sharma. Neuro Fuzzy Based Dynamic Secure Routing Protocol for QoS Frameworks of MANET, International Journal on Emerging Technologies, Volume 10, issue 3, pp. 286-295, 2019.

18. S. Banik, B. Roy, P. Dey, N. Chaki, and S. Sanyal. QoS Routing using OLSR with Optimization for Flood, IJICTR, Volume 1 issue 4, 2016.

19. K. Singh, K. Singh, A. Le, and A. Ahmed. Congestion Control in Wireless Sensor Networks by Hybrid MultiObjective Optimization Algorithm, Computer Networks. 138.10.1016/ j.comnet. 2018.03. 023, 2018.

20. P. Kamboj, and A. K. Sharma. Energy efficient multicast routing protocol for MANET with minimum control overhead (EEMPMO), International Journal of Computer Applications, 8.10.5120/1224-1780, 2010.

21. Castaneda, R. Das, S. Marina, and Mahesh. Query Localization Techniques for On-Demand Routing Protocols in Ad Hoc Networks, Wireless Networks. 8.137-151.10.1023/A: 101371-1808509, 2002.

22. Liao, Z. Wang, J. Zhang, S. Cao, J. Min, and Geyong. Minimizing Movement for Target Coverage and Network Connectivity in Mobile Sensor Networks, Parallel and Distributed Systems, IEEE Transactions on. 26, pp. 1971-1983, 10.1109/TPDS.2014.2333011, 2015.

23. S. Shivashankar, H. N. Varaprasad, and J. Guruswamy. Designing Energy Routing Protocol With Power Consumption Optimization in MANET, Emerging Topics in Computing, IEEE Transactions on. 2, pp. 192197. 10.1109/TETC.2013. 2287177, 2014.

24. F. Alghfari, S. Khalid, and D. Wang. Energy consumption evaluation of AODV and AOMDV routing protocols in mobile Ad-Hoc networks, International Journal of Advanced Computer Science and Applications, 9.10.14569/ IJACSA.2018. 090835, 2018.

25. M. Al-Ghazal, A. El-Sayed, and H. Kelash. Routing Optimization using Genetic Algorithm in Ad-Hoc Networks, pp. 497-503.10.1109/ ISSPIT. 2007. 4458010, 2007.

26. R. Vadivel, and V. Bhaskaran. Energy Efficient With Secured Reliable Routing Protocol (EESRRP) For Mobile Ad-Hoc Networks, Procedia Technology.4, pp703-707. 10.1016/ j.protcy. 2012.05.114, 2012. https://doi.org/10.1016/j.protcy.2012.05.114

27. C. Perkins, E. B. Royer, and S. Das. Ad hoc On-Demand Distance Vector (AODV) Routing, Retrieved March 1, 2001.

28. T. S. Vamsi, P. Kumar, and T. Sruthi. Performance Analysis of AODV Routing Protocol in MANET under Blackhole Attack, Journal of Engineering Research and Application, ISSN: 2248-9622, 9(5), pp. 58-63, 2019.

29. Y. Zheng, H. Ling, and J. Xue. Disaster Rescue Task Scheduling: An Evolutionary Multi-Objective 
Optimization Approach, IEEE Transactions on Emerging Topics in Computing, 6(2), pp. 288-300, 2018.

30. D. Bisen, and S. Sharma. An Enhanced Performance through Agent-based Secure Approach for Mobile Ad Hoc Networks, International Journal of Electronics, 105(1), pp. 14-22, 2017.

31. R. Patel, and P. Kamboj. A Survey on Contemporary MANET Security: Approaches for Securing the MANET, International Journal of Engineering and Technology, 1(9), pp. 98-112, 2017.

32. G. Jagadeesh, J. Vellingiri, J. Gitanjali, K. Arivuselvan, and S. Sudhakar. An Improved Trusted On-Demand Multicast Routing with QoS for Wireless Networks, International Journal of Advanced Trends in Computer Science and Engineering. Volume 9, No.1, Jan. - Feb. 2020.

https://doi.org/10.30534/ijatcse/2020/39912020

33. M. Fotino. and D. R. Floriano. Energy Issues and Energy Aware Routing in Wireless Ad-Hoc Networks, 10.5772/13309, 2011.

34. B. Olagbegi. A Review of the Energy Efficient and Secure Multicast Routing Protocols for Mobile Ad Hoc Networks, International Journal on Applications of Graph Theory in Wireless ad hoc Networks and Sensor Networks. 2.10.5121/jgraphoc. 2010.2201, 2010.

35. G. A. Santhi, and Nachiappan. A Survey of QOS Routing Protocols For Mobile Ad Hoc Networks, International Journal of Computer Science \& Information Technology. 2.10.5121/ ijcsit.2010.2411, 2010.

36. M. Vijaya, and S. Rao. Efficient Multicast Routing Protocol For MANETs, Vol.6, Nov-Dec 2016., 22503501, 2018.

37. P. Kumar, A. Doegar, and B. Kumar. Impact of Mobility on Energy Consumption of AODV Protocol for Routing in Mobile Ad Hoc Networks, International Journal of Computer Applications. 152, pp. 14-17. 10.5120/ ijca2016911836, 2016. 